\title{
2D FLOW MODEL FOR CALCULATING EFFECTIVE AREA OF PISTON-CYLINDER UNITS
}

\author{
W. Sabuga ${ }^{1}$, A. S. Hashad ${ }^{2}$, a S. Ehlers ${ }^{3}$ \\ ${ }^{1}$ Physikalisch-Technische Bundesanstalt (PTB), Braunschweig, Germany, wladimir.sabuga@ptb.de \\ ${ }^{2}$ National Institute for Standards (NIS), Giza, Egypt, ${ }^{\text {a }}$ Guest researcher at PTB, ahmed hashad84@ hotmail.com \\ ${ }^{3}$ Physikalisch-Technische Bundesanstalt (PTB), Braunschweig, Germany, sven.ehlers@ ptb.de
}

\begin{abstract}
:
A 2D flow model is described for calculation of the effective area $(A)$ of pressure-measuring pistoncylinder units (PCU) based on their dimensional properties. With the 2D model, the uncertainty contribution associated with PCU's axial nonsymmetry can be eliminated and the uncertainty of $A$ can be reduced. The $2 \mathrm{D}$ model is applied to several primary PCUs operated in absolute and gauge pressure modes with different pressuretransmitting media. The benefit of the 2D model in dependence on PCU's geometrical perfectness is discussed.
\end{abstract}

Keywords: pressure balance; effective area; dimensional data; flow model

\section{INTRODUCTION}

Determination of $A$ from PCU's dimensional properties has become the main method applied by primary laboratories to realise the pressure unit by means of pressure balances. The uncertainty of this realisation can significantly depend on PCU's geometrical imperfectness, i.e. deviations of the piston and cylinder bore from a perfect cylindrical shape. Axial variations of the piston-cylinder radii can be considered by applying 1D flow models, e.g. such as proposed by Dadson et al. [1]. With the 1D flow models, tangential variations of the PCU radii are addressed by calculating $A$ values for different angular directions and taking their mean and standard deviation. The later can be a significant uncertainty factor in the case of axially nonsymmetrical PCUs. With a 2D flow model, a calculation of $A$ becomes possible which takes into account variations of PCU radii in both, tangential and axial directions. Herewith, a single $A$ value for the whole PCU is obtained, and the uncertainty contribution due to PCU's axial non-symmetry is removed.

\section{THEORY}

In the first approximation, $A$ of a PCU can be taken as a mean cross-sectional area of the piston and cylinder bore. However, due to variability of piston and cylinder bore radii, $r=r(z, \varphi)$ and $R=$ $R(z, \varphi)$, along with PCU's axial and tangential coordinates $Z$ and $\varphi$, the uncertainty of such a determination is relatively high. Variation of $r$ and $R$ with $z$ can be addressed in the $A$ determination by modelling the fluid flow in the axial direction.

\subsection{D flow model}

In this model, the PCU is considered as axisymmetric with a gap profile corresponding to a selected tangential coordinate $\varphi$. For this particular $\varphi$, in the most general case, the effective area can be calculated by [1]:

$A_{\varphi}=\pi r_{0}^{2}\left[1+\frac{h_{0}}{r_{0}}+\frac{1}{r_{0}\left(p_{1}-p_{2}\right)} \int_{0}^{l}(p-\right.$ $\left.\left.p_{2}\right) \frac{\mathrm{d}(r+R)}{\mathrm{d} z} \mathrm{~d} z\right]$,

where $r_{0}=r(0, \varphi), h_{0}=h(0, \varphi), h=h(z, \varphi)=$ $R(z, \varphi)-r(z, \varphi)$, and $p=p(z, \varphi)$ is pressure distribution along the PCU gap having length $l$, and $p_{1}$ and $p_{2}$ are pressures at the gap entrance $(z=0)$ and exit $(z=l)$. Within the 1D flow model, for a selected $\varphi$, pressure distribution $p(z)$ depends on gap width for this $\varphi$ only, on boundary conditions $p_{1}$ and $p_{2}$, as well as on density $\rho=\rho(p)$ and viscosity $\eta=\eta(p)$ of the pressure-transmitting medium in the PCU.

Based on the continuity equation for a 1D flow, in [1], equations for $p(z)$ are derived for incompressible media (typically liquids at moderate pressures) as

$p(z)=p_{1}-\left(p_{1}-p_{2}\right) \frac{\int_{0}^{z} \mathrm{~d} z / h^{3}}{\int_{0}^{l} \mathrm{~d} z / h^{3}}$,

and for media having pressure-proportional density (ideal gas) as 
$p(z)=\left[p_{1}^{2}-\left(p_{1}^{2}-p_{2}^{2}\right) \frac{\int_{0}^{z} \mathrm{~d} z / h^{3}}{\int_{0}^{l} \mathrm{~d} z / h^{3}}\right]^{0.5}$,

both cases for media of constant viscosity. For both cases, expressions for $A$ of oil and of gas pressure balances operated in absolute mode are presented in [1].

When operating a PCU at low pressures, at which molecular properties of the gas require consideration, the pressure distribution can be determined by equation

$p(z)=p_{1}-\left(p_{1}-p_{2}\right) \frac{\int_{0}^{z} \mathrm{~d} z /\left(h^{2} G_{p}\right)}{\int_{0}^{l} \mathrm{~d} z /\left(h^{2} G_{p}\right)}$,

in which $G_{p}$ is the Poiseuille coefficient calculated by methods of the Rarefied Gas Dynamics (RGD) [2].

Having calculated $A_{\varphi}$ for each $\varphi$, the effective area of the whole PCU $\left(A_{1 \mathrm{D}}\right)$ is taken as the mean and its uncertainty due to PCU's axial nonsymmetry ( $u_{\mathrm{ans}}$ ) as the standard deviation of $A_{\varphi}$.

\subsection{D flow model}

Continuity equation for a flow in a 2D gap built by two motionless surfaces is given by equation

$\frac{\partial}{\partial z}\left(h^{3} \frac{\rho}{\eta} \frac{\partial p}{\partial z}\right)+\frac{\partial}{\partial t}\left(h^{3} \frac{\rho}{\eta} \frac{\partial p}{\partial t}\right)=0$,

in which $t$ designates the tangential coordinate, $t=$ $r \varphi$, where $\varphi$ is angular coordinate [1].

For an incompressible medium with a pressureindependent viscosity, $\rho(p)=$ const, $\quad \eta(p)=$ const, it writes as

$\frac{\partial^{2} p}{\partial z^{2}}+\frac{\partial^{2} p}{\partial t^{2}}+\frac{3}{h} \frac{\partial h}{\partial z} \frac{\partial p}{\partial z}+\frac{3}{h} \frac{\partial h}{\partial t} \frac{\partial p}{\partial t}=0$,

and for a compressible medium which obeys the ideal gas law, $\rho(p) \sim p, \eta(p)=$ const, as

$\frac{\partial^{2} p^{2}}{\partial z^{2}}+\frac{\partial^{2} p^{2}}{\partial t^{2}}+\frac{3}{h} \frac{\partial h}{\partial z} \frac{\partial p^{2}}{\partial z}+\frac{3}{h} \frac{\partial h}{\partial t} \frac{\partial p^{2}}{\partial t}=0$.

For 2D pressure distribution $p(z, \varphi)$, the following boundary conditions apply: $p(0, \varphi)=p_{1}$, $p(l, \varphi)=p_{2}, p(z, 0)=p(z, 2 \pi)$. Using these boundary conditions, equations (6) and (7) can be solved numerically by the Finite Difference Method (FDM) as described in [3].

With $p(z, \varphi)$ calculated within the 2D flow model, the effective area $\left(A_{2 \mathrm{D}}\right)$ is obtained by

$$
\begin{aligned}
& A_{2 \mathrm{D}}=\frac{1}{2} \int_{0}^{2 \pi} r_{0}^{2}\left[1+\frac{h_{0}}{r_{0}}+\frac{1}{r_{0}\left(p_{1}-p_{2}\right)} \int_{0}^{l}(p-\right. \\
& \left.\left.p_{2}\right) \frac{\mathrm{d}(r+R)}{\mathrm{d} z} \mathrm{~d} z\right] \mathrm{d} \varphi .
\end{aligned}
$$

Essentially, $A_{2 \mathrm{D}}$ involves the $2 \mathrm{D}$ pressure distribution for the whole axially non-symmetric PCU and, consequently, is free of $u_{\text {ans }}$ existing in the case of the 1D flow modelling.

\section{PISTON-CYLINDER UNITS}

Totally 7 PCUs of different type concerning their size and shape, pressure-transmitting medium and operation mode were studied. All these PCUs were manufactured by DH Instruments, later Fluke Calibration, and made of cobalt- or nickel-bound tungsten carbide. They were characterised dimensionally, are traceable to length standards and thus allow the realisation of the pressure unit as primary pressure standards.

\subsection{Oil-operated 10 MPa PCUs}

Three specially designed PCUs of $5 \mathrm{~cm}^{2}$ nominal effective area are used at PTB as primary standards for the range $10 \mathrm{MPa}$ of gauge oil pressure [4]. They have serial nos. 278, 279, 280 and are operated with di-ethyl-hexyl-sebacate (DEHS) as pressuretransmitting liquid in a modified Harwood twin pressure balance with a maximum load of $500 \mathrm{~kg}$ on each piston. The effective area ratios of any two PCUs could be determined from cross-float experiments with a relative standard uncertainty of about $2 \cdot 10^{-6}$. In [4], the effective areas of the PCUs were determined from their dimensional properties by the Dadson theory for the 1D flow using eqs. (1) and (2), assuming DEHS as an incompressible fluid with a constant viscosity. The theoretical $A$ of the PCUs agreed with the experimental $A$ ratios within $(-5.7$ to 3.8$) \times 10^{-6}$. The relative standard uncertainty of the dimensional $A$ varied between (4.2 and 5.5) $\times 10^{-6}$, with $u_{\text {ans }}=(1.1-3.8) \times 10^{-6}$. This shows a potential for an uncertainty reduction by applying the $2 \mathrm{D}$ flow model.

\subsection{Gas-operated $700 \mathrm{kPa}$ PCUs}

Three PCUs with serial nos. 1159, 1162, 1163 have a nominal effective area of $20 \mathrm{~cm}^{2}$. They were created to be used for pressure measurements in the experiments on the determination of the Boltzmann constant by the Dielectric-Constant Gas Thermometry (DCGT) [3], [5]-[8]. They are operated in two pressure balance platforms, manufactured by Fluke Calibration, equipped with $150 \mathrm{~kg}$ mass sets and automated mass handlers, which allow automated cross-float measurements in absolute and gauge mode [5]. The PCUs have a cylinder-floating design with a double thickness of the cylinder walls, $8 \mathrm{~mm}$, compared with that of the commercial $20 \mathrm{~cm}^{2}$ PCUs. Herewith, the PCU distortion coefficient was reduced, and cylinder roundness was improved. Based on the 1D flow model, the effective area of these PCUs was determined with a relative standard uncertainty, $u(A)=(0.8-1.2) \times 10^{-6}$, which included uncertainty contributions due to PCUs axial non-symmetry.

In the DCGT experiments, these PCAs were operated in absolute pressure mode with helium as a pressure-transmitting medium, but their 
theoretical and experimental characterisation was performed also in gauge mode and with nitrogen as well. Widespread results for $A$ of these PCUs at different conditions were obtained in [3], which include calculation by the 2D flow model in gauge mode based on PCUs' roundness data. With the 2D model, $u(A)$ could be reduced to $(0.5$ to 0.8$) \times 10^{-6}$. In the present work, the analysis is extended to the absolute mode, is performed at several pressures and is based on both, straightness and roundness data of the PCUs as explained later.

\subsection{Gas-operated 15 kPa PCU}

The PCU with the serial no. 183 has a nominal effective area of $9.8 \mathrm{~cm}^{2}$ and is used in a forcebalanced piston gauge, manufactured by Fluke Calibration and known as FPG8601, capable of measuring absolute and gauge pressures in the range $1 \mathrm{~Pa}$ to $15 \mathrm{kPa}$ [9]. The cylinder has a bi-conical bore and two holes in the middle of its length, which are used for piston suspension and gas feed for the PCU lubrication. The lubricant gas flows through the tapered annular gap between the piston and cylinder which centres the piston in the cylinder bore, thus preventing any contact between the piston and the cylinder without piston rotation. The absolute pressure of the lubrication gas has a constant value of $140 \mathrm{kPa}$ in gauge mode and $40 \mathrm{kPa}$ in absolute mode. In [10] and [11], the FPG was characterised as a primary pressure standard using PCU's dimensional properties. In that analyses, the 1D flow model was applied with the pressure distribution calculated using RGD methods. In [10], $u(A)$ was equal to $(9.8$ and 9.4$) \times 10^{-6}$ in absolute and gauge mode, respectively, and was dominated by $u_{\text {ans }}$ being of up to $8.8 \times 10^{-6}$ in dependence on pressure and operation mode. This high $u_{\text {ans }}$ is explained by PCU's big length of about $100 \mathrm{~mm}$, cylinder's biconical shape and a complicated manufacture procedure. The high $u_{\text {ans }}$ indicates a possibility for an uncertainty improvement by its eliminating. Handling the PCU as axisymmetric, $u(A) \leq 1.31 \cdot 10^{-6}$ was obtained in [11] where uncertainty contributions of the PCU diameters and the RGD model were considered.

\section{PCUS DIMENSIONAL PROPERTIES}

Dimensional properties of the pistons and cylinders were determined from measurements of their diameters, straightness and roundness by different measurement techniques and devices. The straightness measurements were performed for 8 generatrix traces separated by $45^{\circ}$. Roundness measurements were done in sections equidistantly distributed by $4 \mathrm{~mm}$ for the $5 \mathrm{~cm}^{2}$ and $20 \mathrm{~cm}^{2}$ PCUs, and by $7 \mathrm{~mm}$ for the $10 \mathrm{~cm}^{2}$ PCU of the FPG.

The diameters, straightness and roundness deviations of the oil-operated $5 \mathrm{~cm}^{2}$ PCUs were measured with standard uncertainties $(k=1)$ of $(25$, 15 and 7.5) nm, respectively [12]. A procedure for linking the diameters and form deviation data was used as described in [12]. The uncertainty of the 3D data produced by the linking contributed $4 \cdot 10^{-6}$ to the relative standard uncertainty of $A$ [4].
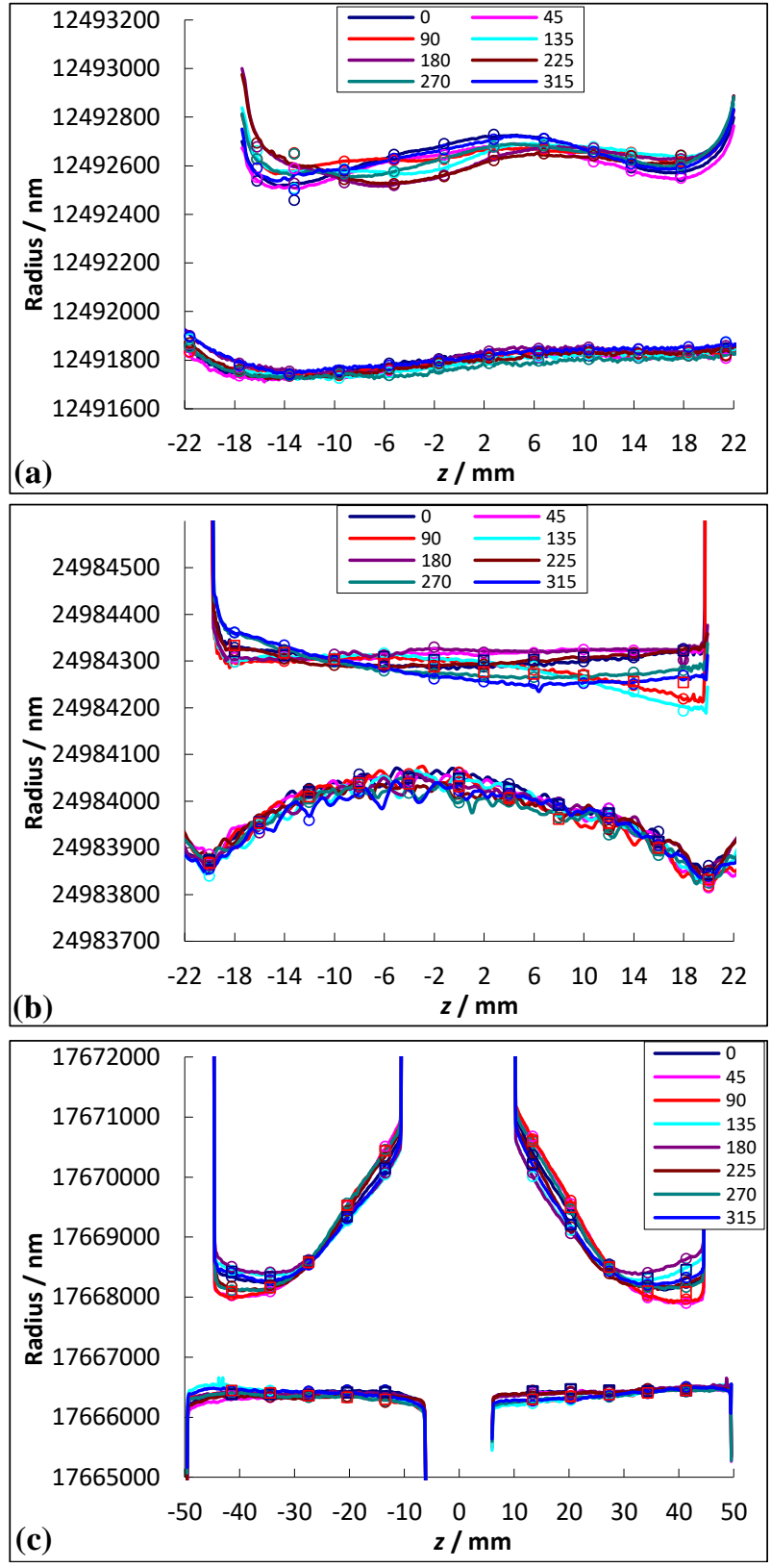

Figure 1: Piston and cylinder radii of PCUs 278, oil, $5 \mathrm{~cm}^{2}$ (a), 1162, gas, $20 \mathrm{~cm}^{2}$ (b), and 183, gas, $10 \mathrm{~cm}^{2}$ (c), along 8 generatrix traces.

In the dimensional measurements of the gasoperated $20 \mathrm{~cm}^{2}$ PCUs, several instruments were involved [13], but the lowest uncertainties were achieved with a modified MarForm MFU8 device for form deviations and a reference length comparator KOMF [14] for diameter measurements, with piston and cylinder diameters measured with the standard uncertainties of $5 \mathrm{~nm}$ and $10 \mathrm{~nm}$, respectively. For linking the diameter and form deviation data, a least-squares (LS) procedure was applied described in [15]. Finally, the radii of the 
piston and cylinders were determined with standard uncertainties from $8 \mathrm{~nm}$ to $19 \mathrm{~nm}$, which contributed ( 0.5 to 0.8$) \cdot 10^{-6}$ to $u(A)$ [6].

For the gas-operated PCU of the FPG8601, the diameters and the form deviations were all measured by the MFU 8 device with the standard uncertainty of diameters of $25 \mathrm{~nm}$. After applying the LS procedure to link the diameter and form deviations data [15], the piston and cylinder radii were obtained with the standard uncertainties of $45 \mathrm{~nm}$ and $31 \mathrm{~nm}$, respectively. Considering the uncertainties of the piston and cylinder radii as noncorrelated, the respective contribution to the relative uncertainty of $A$ was estimated as $3.2 \cdot 10^{-6}[10]$.

Figure 1 shows gap profiles between piston and cylinder for the 3 PCUs of different types. The mean gap widths of the $20 \mathrm{~cm}^{2}$ (gas) and $5 \mathrm{~cm}^{2}$ (oil) PCUs are of about $0.3 \mu \mathrm{m}$ and $0.8 \mu \mathrm{m}$, respectively. The gap width of the $10 \mathrm{~cm}^{2}$ PCU of FPG is much bigger and varies from about $1.5 \mu \mathrm{m}$ to $5 \mu \mathrm{m}$. Also, the FPG PCU shows much bigger variations of the cylinder radius with angle than the classical $20 \mathrm{~cm}^{2}$ and $5 \mathrm{~cm}^{2}$ PCUs. This explains the high uncertainty contribution of $u_{\text {ans }}$ reported in [10].

For getting an optimal accuracy of a 2D pressure distribution by a numerical integration of equations (6) and (7), dimensional data should have a similar density distribution in both axial and tangential directions. The original dimensional data obtained from the straightness measurements has a high density in the axial direction, typically $\geq 10 \mathrm{~mm}^{-1}$, but only (0.05 to 0.1$) \mathrm{mm}^{-1}$ in the tangential direction. Vice versa, the roundness measurement data has a high density in the tangential direction, (2.3 to 4.6$) \mathrm{mm}^{-1}$, but only $(0.14$ to 0.25$) \mathrm{mm}^{-1}$ in the axial direction. Additional dimensional data were generated by a linear interpolation of the straightness radii with the angle step of $1^{\circ}$ and of the roundness radii with the axial step of $0.1 \mathrm{~mm}$. These data sets had similar density distributions of (2.3 to 10 ) $\mathrm{mm}^{-1}$ in both directions. Finally, two data sets with the same structure were obtained based on the straightness and roundness data, and, using them, all calculations were performed two times.

\section{MODELLING CONDITIONS}

For the classical, oil-operated $5 \mathrm{~cm}^{2}$ and gasoperated $20 \mathrm{~cm}^{2}$ PCUs, the calculations were performed at $10 \%$ and $100 \%$ of the PCUs' pressure operation range, for the $20 \mathrm{~cm}^{2}$ PCUs in both gauge and absolute pressure mode. As a boundary condition at the PCU exit, $p_{2}=100 \mathrm{kPa}$ in gauge mode and $p_{2}=0.1 \mathrm{~Pa}$ in absolute mode was used.

For the $10 \mathrm{~cm}^{2}$ PCU of the FPG, separate modelling of the PCU's upper and lower parts was performed, because, due to the lubrication pressure in the middle of the PCU, each part has its own boundary conditions [10]. As $p_{1}, 40 \mathrm{kPa}$ was taken for the absolute and $140 \mathrm{kPa}$ for the gauge mode in the modelling of both PCU parts. As $p_{2}, 0.5 \mathrm{~Pa}$ was
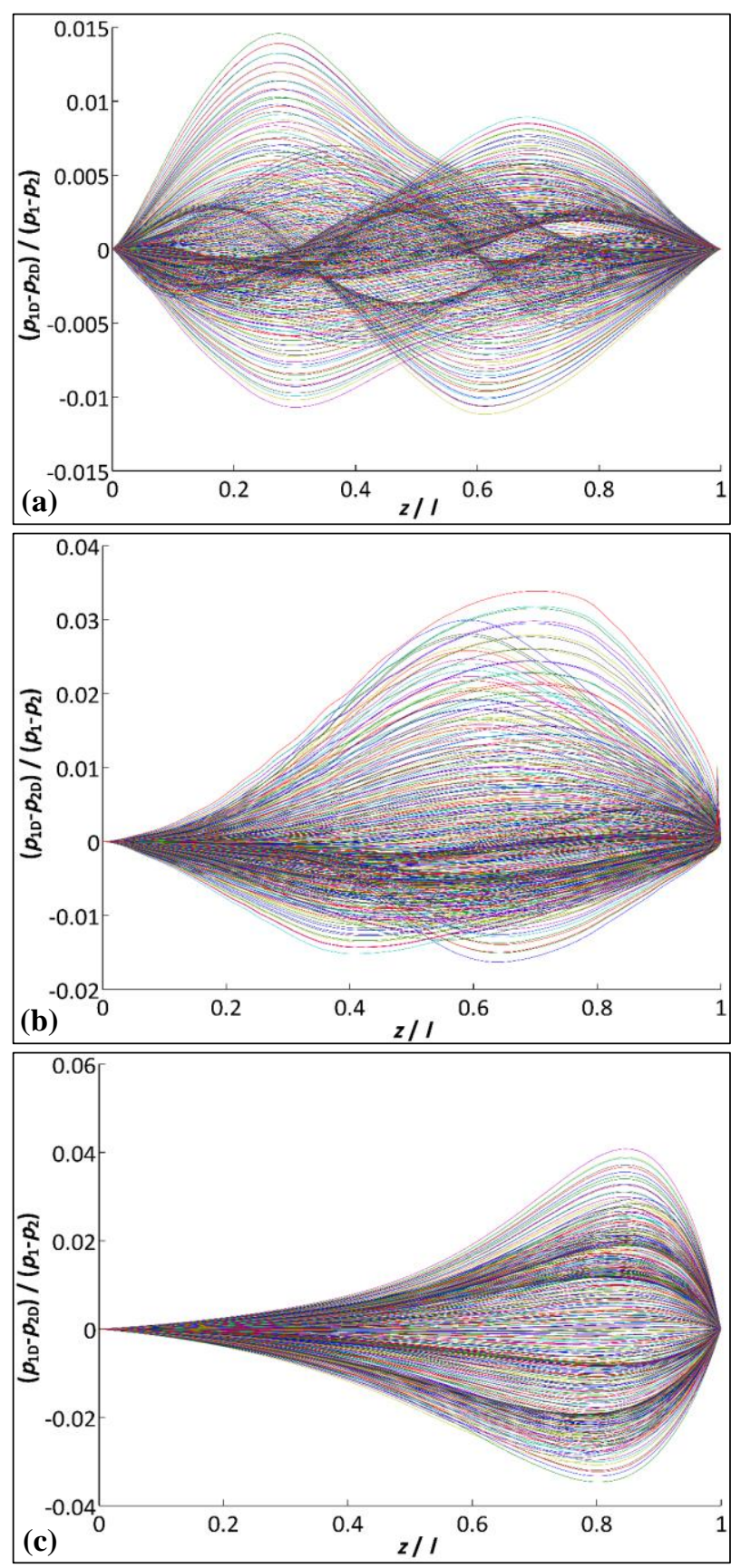

Figure 2: Relative difference of $1 \mathrm{D}$ and 2D pressure distributions in the gap of PCUs 278, oil, gauge mode, $p_{1}=10 \mathrm{MPa}$ (a); 1162, gas, absolute mode, $p_{1}=700 \mathrm{kPa}$ (b); and upper part of PCU 183, gas, absolute mode, $p_{1}=40 \mathrm{kPa}, p_{2}=0.5 \mathrm{~Pa}(\mathrm{c})$, all based on straightness data.

taken for the absolute and $100 \mathrm{kPa}$ for the gauge mode in the modelling of the lower PCU part. For the upper part, $p_{2}$ was taken as $1 \mathrm{~Pa}$ and $15 \mathrm{kPa}$ for the absolute mode as well as $100001 \mathrm{~Pa}$ and $115 \mathrm{kPa}$ in the gauge mode, which corresponds to the minimum and maximum pressure measured by the FPG in the two pressure modes.

First, 1D flow pressure distribution $p(z)$ was calculated for a PCU gap profile of each $\varphi=0,1$, $\ldots, 359^{\circ}$. The calculation was done using equation (2) for the oil-operated and (3) for gas-operated 
PCUs. Then, taking this 1D flow pressure distribution as a starting condition, a 2D pressure distribution $p(z, \varphi)$ was searched iteratively by the FDM. The iterations were repeated until the maximum pressure difference at any point $(z, \varphi)$ in two subsequent iterations became smaller than $10^{-7} \times\left(p_{1}-p_{2}\right)$.

\section{RESULTS}

Results of the 2D pressure distribution calculations for 3 PCUs of different types are presented in Figure 2 in relation to the 1D pressure distribution. They show deviations of the pressure distribution along 360 generatrix lines of the 2D flow model from the pressure distribution of the 1D flow model obtained for each gap profile. The difference between the $1 \mathrm{D}$ and $2 \mathrm{D}$ pressure distributions is bigger in the case of compressible fluid (gas) and the absolute pressure mode (Figure 2, $\mathrm{b}$ and c) than in the case of incompressible fluid (oil) and gauge mode (Figure 2, a).

The difference is bigger for the upper part of the FPG PCU 183 (c) than for the classical PCU 1162 (b) because the first is stronger axially nonsymmetric than the second one. Moreover, the maximum of the pressure distributions' deviation in the case of the FPG PCU is shifted to the gap exit $(z \rightarrow l)$, evidently, because of the pronounced gap narrowing towards the gap exit.

Results of $A$ calculation for the oil-operated PCUs are shown in Table 1 and for the gas-operated PCUs in Table 2. The values of $A$ and $u_{\text {ans }}$ in the tables are those obtained for the maximum difference of $p_{1}$ and $p_{2}$, but the results at smaller $p_{1}-p_{2}$ were very close to the values in the tables. The variation of the values was taken into account when calculating mean $A$ and $u_{\text {ans. }}$. For the 1D flow model, $u_{\text {ans }}$ was calculated as explained in section 2.1. For the $2 \mathrm{D}$ model, $u_{\text {ans }}$ was defined as the difference of $A$ values obtained by the 1D and 2D flow models. 1D $u_{\text {ans }}$ varies between $(0.83$ and $3.33) \times 10^{-6}$, and, thus, for PCU 280 is comparable with the uncertainty of the dimensional measurements. By applying the 2D model, $u_{\mathrm{ans}}$ is reduced to below $0.07 \cdot 10^{-6}$, and the combined relative standard uncertainties become lower than those of the 1D flow model. For all 3 PCUs, they are equal to $4 \cdot 10^{-6}$ and are slightly lower than reported in [4].

For gas-operated PCUs, the results on $u_{\text {ans }}$ obtained for the gauge mode were similar with those for the absolute mode. For the $20 \mathrm{~cm}^{2}$ PCUs, 1D $u_{\text {ans }}$ varies between $(0.49$ and 1.1$) \times 10^{-6}$, and therefore is comparable with the dimensional uncertainties. By applying the 2D model, $u_{\text {ans }}$ is reduced to values below $0.03 \cdot 10^{-6}$, which agrees with the results previously obtained for these PCUs in gauge mode using $\mathrm{R}$ data in [3]. Finally, the combined relative standard uncertainties are essentially lower with the 2D flow than with the 1D flow model. For the FPG PCU 183, whose dimensional irregularities are much bigger than those of the $20 \mathrm{~cm}^{2}$ PCUs, 1D $u_{\text {ans }}$ strongly dominates the uncertainty budget with a contribution of about $10 \cdot 10^{-6}$. By applying the 2D model it is reduced to $0.15 \cdot 10^{-6}$, which significantly reduces the combined uncertainty to about $3.2 \cdot 10^{-6}$. In [10], a standard uncertainty of $9.8 \cdot 10^{-6}$ was reported for the absolute mode which was affected by the axial non-symmetry of the $\mathrm{S}$ data used in the calculation.

Table 1: Effective area $(A)$ of oil-operated PCUs 278, 279 and 280 at gauge pressure $p_{1}-p_{2}=10 \mathrm{MPa}$, calculated on the basis of straightness $(\mathrm{S})$ and roundness $(\mathrm{R})$ data using 1D and 2D flow models; uncertainties due to PCUs' axial nonsymmetry $\left(u_{\mathrm{ans}}\right)$, dimensional data $\left(u_{\mathrm{dim}}\right)$, variation of $A$ with pressure and choice of $\mathrm{S}$ or $\mathrm{R}$ data, and combined uncertainty $\left(u_{\mathrm{comb}}\right)$, all uncertainties being relative standard ones in $10^{-6} .\langle A\rangle$ and $\left\langle u_{\text {ans }}\right\rangle$ are means of $A$ and $u_{\text {ans }}$ at $p_{1}-$ $p_{2}=(1$ and 10) MPa.

\begin{tabular}{|c|c|c|c|c|c|c|c|}
\hline \multirow{2}{*}{\multicolumn{2}{|c|}{ Data, Model }} & \multicolumn{2}{|c|}{278} & \multicolumn{2}{|c|}{279} & \multicolumn{2}{|c|}{280} \\
\hline & & \multirow{2}{*}{$\begin{array}{l}\boldsymbol{A} / \mathbf{m m}^{\mathbf{2}} \\
490.2620\end{array}$} & \multirow{2}{*}{$\begin{array}{c}\boldsymbol{u}_{\text {ans }} \\
1.15 \\
\end{array}$} & \multirow{2}{*}{$\frac{\boldsymbol{A} / \mathbf{~ m m}^{\mathbf{2}}}{490.2634}$} & \multirow{2}{*}{$\frac{\boldsymbol{u}_{\text {ans }}}{0.59}$} & \multirow{2}{*}{$\frac{\boldsymbol{A} / \mathbf{~ m m}^{\mathbf{2}}}{490.2648}$} & \multirow{2}{*}{$\frac{\boldsymbol{u}_{\text {ans }}}{3.07}$} \\
\hline \multirow{2}{*}{$\mathrm{S}$} & $1 \mathrm{D}$ & & & & & & \\
\hline & $2 \mathrm{D}$ & 490.2620 & 0.02 & 490.2634 & 0.01 & 490.2648 & 0.02 \\
\hline \multirow{2}{*}{$\mathrm{R}$} & $1 \mathrm{D}$ & 490.2618 & 1.18 & 490.2633 & 1.07 & 490.2647 & 3.59 \\
\hline & $2 \mathrm{D}$ & 490.2618 & 0.09 & 490.2633 & 0.01 & 490.2647 & 0.01 \\
\hline \multicolumn{2}{|c|}{$\langle A\rangle / \mathrm{mm}^{2}$} & \multicolumn{2}{|c|}{490.2619} & \multicolumn{2}{|c|}{490.2633} & \multicolumn{2}{|c|}{490.2648} \\
\hline \multicolumn{2}{|c|}{$u_{\text {dim }}$} & \multicolumn{2}{|c|}{4.00} & \multicolumn{2}{|c|}{4.00} & \multicolumn{2}{|c|}{4.00} \\
\hline \multicolumn{2}{|c|}{$\Delta_{A}$} & \multicolumn{2}{|c|}{0.13} & \multicolumn{2}{|c|}{0.08} & \multicolumn{2}{|c|}{0.04} \\
\hline \multirow{2}{*}{$\left\langle u_{\text {ans }}\right\rangle$} & $1 \mathrm{D}$ & \multicolumn{2}{|c|}{1.16} & \multicolumn{2}{|c|}{0.83} & \multicolumn{2}{|c|}{3.33} \\
\hline & $2 \mathrm{D}$ & \multicolumn{2}{|c|}{0.07} & \multicolumn{2}{|c|}{0.01} & \multicolumn{2}{|c|}{0.01} \\
\hline \multirow{2}{*}{$u_{\text {comb }}$} & $1 \mathrm{D}$ & \multicolumn{2}{|c|}{4.17} & \multicolumn{2}{|c|}{4.09} & \multicolumn{2}{|c|}{5.20} \\
\hline & $2 \mathrm{D}$ & \multicolumn{2}{|c|}{4.00} & \multicolumn{2}{|c|}{4.00} & \multicolumn{2}{|c|}{4.00} \\
\hline
\end{tabular}


Table 2: Effective area of gas-operated PCUs at pressures $p_{1}=700 \mathrm{kPa}$ and $p_{2}=0.1 \mathrm{~Pa}$ for PCUs 1159,1162 and 1163 , and $p_{1}=40 \mathrm{kPa}$ and $p_{2}=1 \mathrm{~Pa}$ for the upper part of PCU 183. $\langle A\rangle$ and $\left\langle u_{\text {ans }}\right\rangle$ present results at $p_{1}=(70$ and $700 \mathrm{kPa})$ for PCUs 1159, 1162 and 1163, as well as $\mathrm{d} p_{2}=1 \mathrm{~Pa}$ and $15 \mathrm{kPa}$ for PCU 183. All designations are as in Table 1.

\begin{tabular}{|c|c|c|c|c|c|c|c|c|c|}
\hline \multirow{2}{*}{\multicolumn{2}{|c|}{ Data, Model }} & \multicolumn{2}{|c|}{1159} & \multicolumn{2}{|c|}{1162} & \multicolumn{2}{|c|}{1163} & \multicolumn{2}{|c|}{ 183, upper part } \\
\hline & & $A / \mathrm{mm}^{2}$ & $\boldsymbol{u}_{\text {ans }}$ & $A / \mathrm{mm}^{2}$ & $\boldsymbol{u}_{\text {ans }}$ & $A / \mathrm{mm}^{2}$ & $u_{\text {ans }}$ & $A / \mathrm{mm}^{2}$ & $u_{\text {ans }}$ \\
\hline \multirow{2}{*}{ S } & 1D & 1961.0094 & 0.67 & 1961.0044 & 1.03 & 1961.0423 & 0.35 & 980.6067 & 9.52 \\
\hline & $2 \mathrm{D}$ & 1961.0094 & 0.00 & 1961.0043 & 0.04 & 1961.0422 & 0.03 & 980.6068 & 0.09 \\
\hline \multirow{2}{*}{$\mathrm{R}$} & 1D & 1961.0089 & 0.82 & 1961.0033 & 1.20 & 1961.0411 & 0.63 & 980.6063 & 11.50 \\
\hline & $2 \mathrm{D}$ & 1961.0089 & 0.00 & 1961.0033 & 0.02 & 1961.0411 & 0.01 & 980.6065 & 0.14 \\
\hline \multicolumn{2}{|c|}{$\langle A\rangle / \mathrm{mm}^{2}$} & \multicolumn{2}{|c|}{1961.0092} & \multicolumn{2}{|c|}{1961.0038} & \multicolumn{2}{|c|}{1961.0417} & \multicolumn{2}{|c|}{980.6067} \\
\hline \multicolumn{2}{|c|}{$u_{\text {dim }}$} & \multicolumn{2}{|c|}{0.75} & \multicolumn{2}{|c|}{0.50} & \multicolumn{2}{|c|}{0.65} & \multicolumn{2}{|c|}{3.20} \\
\hline \multicolumn{2}{|c|}{$\Delta_{A}$} & \multicolumn{2}{|c|}{0.08} & \multicolumn{2}{|c|}{0.16} & \multicolumn{2}{|c|}{0.17} & \multicolumn{2}{|c|}{0.27} \\
\hline \multirow{2}{*}{$\left\langle u_{\text {ans }}\right\rangle$} & 1D & \multicolumn{2}{|c|}{0.75} & \multicolumn{2}{|c|}{1.12} & \multicolumn{2}{|c|}{0.49} & \multicolumn{2}{|c|}{10.04} \\
\hline & $2 \mathrm{D}$ & \multicolumn{2}{|c|}{0.00} & \multicolumn{2}{|c|}{0.03} & \multicolumn{2}{|c|}{0.02} & \multicolumn{2}{|c|}{0.15} \\
\hline \multirow[b]{2}{*}{$u_{\text {comb }}$} & $1 \mathrm{D}$ & \multicolumn{2}{|c|}{1.06} & \multicolumn{2}{|c|}{1.23} & \multicolumn{2}{|c|}{0.83} & \multicolumn{2}{|c|}{10.54} \\
\hline & $2 \mathrm{D}$ & \multicolumn{2}{|c|}{0.75} & \multicolumn{2}{|c|}{0.52} & 0.67 & & 3.2 & \\
\hline
\end{tabular}

\section{SUMMARY}

By applying a 2D flow model, the effective area of 3 oil and 4 gas PCUs used as primary standards for gauge and absolute pressures was determined. With this 2D flow model, the uncertainty contribution due to axial non-symmetry of the PCUs' dimensional properties was eliminated and, finally, the combined uncertainty reduced by $2 \%$ to $70 \%$.

\section{REFERENCES}

[1] R. S. Dadson, S. L. Lewis, G. N. Peggs, The pressure balance - Theory and practice, London, HMSO, 1982.

[2] W. Sabuga, F. Sharipov, T. Priruenrom, "Determination of the effective area of pistoncylinder assemblies using a rarefied gas flow model", PTB-Mitteilungen, vol. 121, no. 3, pp. 260-262, 2011.

[3] T. Priruenrom, "Development of pressure balances for absolute pressure measurement in gases up to $7 \mathrm{MPa}$ ", Doctoral thesis, Clausthal University of Technology, Germany, 2011.

[4] J. Jäger, W. Sabuga, D. Wassmann, "Pistoncylinder assemblies of $5 \mathrm{~cm} 2$ cross-sectional area used in an oil-operated primary pressure balance standard for the $10 \mathrm{MPa}$ range", Metrologia, vol. 36, pp. 541-544, 1999.

[5] W. Sabuga, T. Priruenrom, R. Haines, M. Bair, "Design and evaluation of pressure balances with $1 \cdot 10^{-6}$ uncertainty for the Boltzmann constant project", PTB-Mitteilungen, vol. 121, no. 3, pp. 256-259, 2011.

[6] W. Sabuga W., "Pressure measurements in gas media up to $7.5 \mathrm{MPa}$ for the Boltzmann constant redetermination", PTB-Mitteilungen, vol. 121, no. 3, pp. 247-255, 2011.

[7] T. Zandt, W. Sabuga, C. Gaiser, B. Fellmuth, "Measurement of pressures up to $7 \mathrm{MPa}$ applying pressure balances for dielectric-constant gas thermometry", Metrologia, vol. 52, pp. S305-S313, 2015.

[8] C. Gaiser, et al., "Final determination of the Boltzmann constant by dielectric-constant gas thermometry", Metrologia, vol. 54, pp. 280-289, 2017.

[9] P. Delajoud, M. Girard, "A force balanced piston gauge for very low gauge and absolute pressure", Proc. of $10^{\text {th }}$ Intern. Metrology Congress, St Louis, France, 22-25 October 2001.

[10] A. S. Hashad, S. Ehlers, O. Jusko, W. Sabuga, "Characterization of a force-balanced piston gauge as a primary pressure standard", Measurement, vol. 131, pp. 723-729, 2019.

[11] S. Naris, et al., "Computation of the effective area and associated uncertainties of non-rotating piston gauges FPG and FRS”, Metrologia, vol. 56, pp. 110, 2019.

[12] O. Jusko, H. Bosse, F. Lüdicke, "High precision 3D-calibration of cylindrical standards", in Advanced Mathematical Tools in Metrology III, edited by P. Ciarlini, M. G. Cox, F. Pavese and D. Richter, Singapore, World Scientific Publishing Company, 1997, pp. 186-194.

[13] O. Jusko, et al., "Dimensional calibration techniques for pressure for pressure balances to be used in the new determination of the Boltzmann constant", Congresso Internacional de Metrologia Mecanica: Anais, I-CIMMEC, Infosim Informative Bulletin of the Interamerican Metrology System - OAS, August 2009, pp. 14-23.

[14] M. Neugebauer, et al., "A new comparator for measurement of diameter and form of cylinders, spheres and cubes under clean-room conditions", Meas. Sci. Technol., vol. 8, pp. 849-856, 1997.

[15] W. Sabuga, T. Priruenrom, "An approach to the evaluation of dimensional measurements on pressure-measuring piston-cylinder assemblies", Proc. of $3^{\text {rd }}$ Conf. on Pressure Measurement IMEKO TC16, Merida, Mexico, 2007, http://www.imeko.org/publications/tc162007/IMEKO-TC16-2007-074u.pdf. 